\title{
Neurostimulation for Parkinson's Disease with Early Motor Complications
}

\author{
W.M.M. Schuepbach, J. Rau, K. Knudsen, J. Volkmann, P. Krack, L. Timmermann, \\ T.D. Hälbig, H. Hesekamp, S.M. Navarro, N. Meier, D. Falk, M. Mehdorn, S. Paschen, \\ M. Maarouf, M.T. Barbe, G.R. Fink, A. Kupsch, D. Gruber, G.-H. Schneider, E. Seigneuret, \\ A. Kistner, P. Chaynes, F. Ory-Magne, C. Brefel Courbon, J. Vesper, A. Schnitzler, \\ L. Wojtecki, J.-L. Houeto, B. Bataille, D. Maltête, P. Damier, S. Raoul, F. Sixel-Doering, \\ D. Hellwig, A. Gharabaghi, R. Krüger, M.O. Pinsker, F. Amtage, J.-M. Régis, T. Witjas, \\ S. Thobois, P. Mertens, M. Kloss, A. Hartmann, W.H. Oertel, B. Post, H. Speelman, \\ Y. Agid, C. Schade-Brittinger, and G. Deuschl, for the EARLYSTIM Study Group*
}

ABSTRACT

The authors' full names, degrees, and affiliations are listed in the Appendix. Address reprint requests to $D r$. Deuschl at the Department of Neurology, University Hospital Schleswig-Holstein, Campus Kiel, Christian-Albrechts University Kiel, ArnoldHeller Str. 3, 24105 Kiel, Germany, or at g.deuschl@neurologie.uni-kiel.de.

Dr. Schuepbach, Mr. Rau, and Dr. Knudsen and Dr. Agid, Ms. Schade-Brittinger, and Dr. Deuschl contributed equally to this article.

*Additional investigators in the Controlled Trial of Deep Brain Stimulation in Early Patients with Parkinson's Disease (EARLYSTIM) Study Group are listed in the Supplementary Appendix, available at NEJM.org.

N EnglJ Med 2013;368:610-22. DOI: 10.1056/NEJMoal205158 Copyright (c) 2013 Massachusetts Medical Society.

\section{BACKGROUND}

Subthalamic stimulation reduces motor disability and improves quality of life in patients with advanced Parkinson's disease who have severe levodopa-induced motor complications. We hypothesized that neurostimulation would be beneficial at an earlier stage of Parkinson's disease.

METHODS

In this 2-year trial, we randomly assigned 251 patients with Parkinson's disease and early motor complications (mean age, 52 years; mean duration of disease, 7.5 years) to undergo neurostimulation plus medical therapy or medical therapy alone. The primary end point was quality of life, as assessed with the use of the Parkinson's Disease Questionnaire (PDQ-39) summary index (with scores ranging from 0 to 100 and higher scores indicating worse function). Major secondary outcomes included parkinsonian motor disability, activities of daily living, levodopa-induced motor complications (as assessed with the use of the Unified Parkinson's Disease Rating Scale, parts III, II, and IV, respectively), and time with good mobility and no dyskinesia.

\section{RESULTS}

For the primary outcome of quality of life, the mean score for the neurostimulation group improved by 7.8 points, and that for the medical-therapy group worsened by 0.2 points (between-group difference in mean change from baseline to 2 years, 8.0 points; $\mathrm{P}=0.002)$. Neurostimulation was superior to medical therapy with respect to motor disability $(\mathrm{P}<0.001)$, activities of daily living $(\mathrm{P}<0.001)$, levodopa-induced motor complications $(\mathrm{P}<0.001)$, and time with good mobility and no dyskinesia $(\mathrm{P}=0.01)$. Serious adverse events occurred in $54.8 \%$ of the patients in the neurostimulation group and in $44.1 \%$ of those in the medical-therapy group. Serious adverse events related to surgical implantation or the neurostimulation device occurred in $17.7 \%$ of patients. An expert panel confirmed that medical therapy was consistent with practice guidelines for $96.8 \%$ of the patients in the neurostimulation group and for $94.5 \%$ of those in the medical-therapy group.

CONCLUSIONS

Subthalamic stimulation was superior to medical therapy in patients with Parkinson's disease and early motor complications. (Funded by the German Ministry of Research and others; EARLYSTIM ClinicalTrials.gov number, NCT00354133.) 


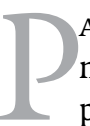
ARKINSON'S DISEASE IS A PROGRESSIVE neurodegenerative disease that affects dopaminergic neurotransmission, resulting in bradykinesia, rigidity, and rest tremor. After an initial honeymoon period, during which there is a sustained response to dopaminergic treatment, beneficial effects are hampered by levodopainduced motor complications, ${ }^{1}$ progressively compromising quality of life. ${ }^{2-4}$

Because levodopa-responsive parkinsonian symptoms are improved by high-frequency stimulation of the subthalamic nucleus, 5,6 neurostimulation has become an established treatment for advanced Parkinson's disease with medically intractable fluctuations and dyskinesia ${ }^{7-10}$ and has shown long-term efficacy. ${ }^{11-13}$ It is typically used after the disease has been present for 11 to 13 years, ${ }^{7-10}$ when quality of life, social adjustment (psychosocial competence), ${ }^{14}$ and professional activity are already severely impaired. Neurostimulation improves quality of life, ${ }^{7-10}$ in addition to motor symptoms. Moreover, later in the course of the disease, features unresponsive to dopaminergic treatment often predominate. Therefore, optimizing quality of life during the period when patients have the greatest response to dopaminergic therapy (and therefore neurostimulation as well) should be considered a major goal of current treatment. We hypothesized that neurostimulation improves quality of life at an earlier stage of Parkinson's disease, as suggested by our pilot trial involving patients with early and mild motor complications. ${ }^{15}$

In the current study, we randomly assigned patients with Parkinson's disease and a recent onset of motor complications to receive neurostimulation plus medical therapy or medical therapy only. Disease-related quality of life was chosen as the primary outcome, thereby allowing a global assessment of beneficial and adverse effects in a way that subjectively matters to the patient.

\section{METHODS}

\section{PATIENTS}

Patients with Parkinson's disease were eligible for the study if they met the following inclusion criteria: an age of 18 to 60 years; disease duration of 4 years or more; a disease severity rating below stage 3 in the on-medication condition, according to the Hoehn and Yahr scale, with scores ranging from 0 to 5 and higher scores indicating more severe disease $\mathrm{e}^{16}$; improvement of motor signs of $50 \%$ or more with dopaminergic medication, ${ }^{6}$ as assessed with the use of the Unified Parkinson's Disease Rating Scale, part III (UPDRS-III; scores range from 0 to 108 , with higher scores indicating worse functioning $)^{17}$; fluctuations or dyskinesia present for 3 years or less; and a score of more than 6 for activities of daily living in the worst condition despite medical treatment, as assessed with the use of the UPDRS-II (scores range from 0 to 52, with higher scores indicating worse functioning), or mild-to-moderate impairment in social and occupational functioning (score of 51 to $80 \%$ on the Social and Occupational Functioning Assessment Scale, ${ }^{18}$ with scores ranging from 1 to 100 and lower scores indicating worse functioning).

Exclusion criteria were dementia (a score of $\leq 130$ on the Mattis Dementia Rating Scale, ${ }^{19}$ with scores ranging from 0 to 144 and higher scores indicating better functioning), major depression with suicidal thoughts (a score of $>25$ on the Beck Depression Inventory II, ${ }^{20}$ with scores ranging from 0 to 63 and higher scores indicating worse functioning), acute psychosis, and any medical or psychological problem that would interfere with the conduction of the study protocol. ${ }^{21}$ Patients with a duration of disease of less than 4 years were excluded because atypical forms of parkinsonism would be expected to be identifiable before then. Details regarding the scales are provided in Tables S1, S2, and S3 in the Supplementary Appendix, available with the full text of this article at NEJM.org.

\section{STUDY DESIGN}

Conducted in Germany and France, this study followed an investigator-initiated, randomized, multicenter, parallel-group design comparing neurostimulation plus medical therapy (neurostimulation group) with medical therapy alone (medical-therapy group). Randomization was performed centrally at the University of Marburg coordinating center, Marburg, Germany, with the use of randomization lists with randomly permuted block lengths stratified according to center.

The trial conformed to the Declaration of Helsinki, Good Clinical Practice guidelines, and the International Organization for Standardization 14:155 (2003) standards and was approved by the ethics committee for each participating center. 
All patients provided written informed consent before randomization. An independent data and safety monitoring committee provided review and direction regarding the collection of safety data.

Full source-data verification was performed by monitors from the German coordinating center (Koordinierungszentrum für Klinische Studien) for the German centers and by monitors from the French coordinating center (Department of Clinical Research, Assistance Publique-Hôpitaux de Paris) for the French centers. Data were collected and analyzed by the German coordinating center in Marburg. All the authors vouch for the accuracy of the data and the analyses reported and for the adherence of the study to the protocol, available at NEJM.org. The protocol committee designed the study, and the steering committee wrote the first draft of the manuscript. Medtronic provided additional funding for the study but had no role in the study design, data accrual, data analysis, or manuscript preparation.

Patients assigned to neurostimulation underwent bilateral stereotactic surgery of the subthalamic nucleus with the implantation of electrodes (model 3389, Medtronic) and a pulse generator (Kinetra or Soletra, Medtronic) within 6 weeks after randomization, according to operative standards that address local anesthesia, imaging, targeting, microelectrode recording, and confirmation of the final electrode position. ${ }^{21}$ Patients in the neurostimulation group then began receiving stimulation according to standards established for this study (see the Supplementary Appendix). ${ }^{21}$

Assessments were scheduled at baseline and at 5,12 , and 24 months. A levodopa challenge test was performed at baseline and at 24 months (see the Supplementary Appendix). ${ }^{21}$ Blinded assessments were based on preoperative and postoperative standardized video recordings obtained at baseline and at 24 months. Videos were recorded for each motor condition (according to whether the patient was or was not receiving medication or stimulation). The UPDRS-III score was assessed by two expert raters who were unaware of the study assignments, ${ }^{22}$ except for the assessment of rigidity, which cannot be evaluated on the basis of a video recording.

During follow-up, adjustments to medication and stimulation were performed according to predefined standards. ${ }^{21}$ These standards followed the European Federation of Neurological Societies guidelines for the treatment of advanced Parkinson's disease ${ }^{23}$ and a standardized sequence of interventions. ${ }^{21}$ An independent expert panel assessed whether medication therapy was consistent with guidelines for each patient (see the Supplementary Appendix).

A specific procedure for monitoring the risk of suicidality, established after two suicides had occurred during the study, consisted of a baseline assessment of the general risk and a semistructured telephone interview every 2 months to assess status, with psychiatric follow-up as needed (see the Supplementary Appendix). ${ }^{21}$

\section{OUTCOME MEASURES}

The primary end point was the between-group difference in mean change in quality of life from baseline to 2 years, as assessed with the use of the summary index of the Parkinson's Disease Questionnaire (PDQ-39).24,25 After we obtained a significant result for quality of life, the following clinically relevant motor functions were tested sequentially as major secondary outcomes: activities of daily living (UPDRS-II score), ${ }^{17}$ severity of motor signs (UPDRS-III score), severity of treatment-related complications (UPDRS-IV score), and time with good mobility and no troublesome dyskinesia, as recorded by patients in a diary. Minor secondary outcomes included scores on the Scales for Outcomes in Parkinson's Disease-Psychosocial (SCOPA-PS) questionnaire (on a scale from 0 to 33, with higher scores indicating worse functioning), the Mattis Dementia Rating Scale ${ }^{19}$ (on a scale of 0 to 144, with higher scores indicating better functioning), the Brief Psychiatric Rating Scale $^{26}$ (on a scale of 18 to 126 , with higher scores indicating worse functioning), the Montgomery and Åsberg Depression Rating Scale ${ }^{27}$ (on a scale of 0 to 60 , with higher scores indicating worse functioning), the Beck Depression Inventory $\mathrm{II}^{20}$ (on a scale of 0 to 63 , with higher scores indicating worse functioning), and the Starkstein Apathy Scale ${ }^{28}$ (on a scale of 0 to 42 , with higher scores indicating worse functioning), as well as the levodopa-equivalent daily dose.,7,21,29 The other minor secondary outcomes are listed in the Supplementary Appendix.

\section{ADVERSE EVENTS}

Adverse events in all patients were reported and coded according to the Medical Dictionary for Regulatory Activities, version 14.1. Serious adverse events were defined as any events that led to death, disability, or prolonged or new hospitalization with serious health impairment. 


\section{STATISTICAL ANALYSIS}

The sample size was calculated on the basis of our previous studies. ${ }^{7,15}$ We selected a power of $80 \%$ for a two-sided Mann-Whitney test, assuming normally distributed data. To detect a standardized effect size of 0.4 with an alpha level of $5 \%$, we calculated that we would need to enroll 208 patients. Assuming a 15\% rate of loss to follow-up, we determined that a total enrollment of at least 246 patients would be required. The intention-to-treat analysis was primary; the perprotocol analysis was secondary. No interim analysis was planned. The Mann-Whitney test was replaced by a flexible and robust linear mixed-model analysis with baseline adjustment and included study center as a random effect, main effects for group and time, a group-by-time interaction term, and a generalized covariance matrix to account for serial dependency among observations after verification of the assumption of multivariate normality for the parametric model. ${ }^{21}$ Differences in mean changes between assessments at baseline and at 24 months were compared between the groups. Missing data due to loss to follow-up were handled by means of direct likelihood analyses, ${ }^{30,31}$ with adjustment for the conditional expectation of the missing measurements, given the observed ones.

A serial gatekeeper procedure was planned. If the primary end point was significant, the UPDRSII, UPDRS-III, and UPDRS-IV scores and time with good mobility and no troublesome dyskinesia were tested sequentially with the use of Hochberg's multiple-comparison method ${ }^{32}$ at a significance level of $5 \%$.

\section{RESULTS}

\section{CHARACTERISTICS OF THE PATIENTS}

Of 392 patients assessed for eligibility, 251 were enrolled between July 2006 and November 2009 at nine German and eight French university centers (Fig. S1 in the Supplementary Appendix). The intention-to-treat population consisted of 124 patients assigned to the neurostimulation group (120 of whom underwent implantation and completed the study) and 127 patients assigned to the medical-therapy group (of whom 125 underwent medical therapy and 123 completed the study). A total of 25 patients had major protocol deviations, including PDQ-39 assessment outside the predefined time window, an absence of motor fluctuations or dyskinesia, insufficient exposure

\begin{tabular}{|c|c|c|}
\hline Characteristic & $\begin{array}{l}\text { Neurostimulation } \\
(\mathrm{N}=124)\end{array}$ & $\begin{array}{l}\text { Medical Therapy } \\
(\mathrm{N}=127)\end{array}$ \\
\hline Age $-y r$ & $52.9 \pm 6.6$ & $52.2 \pm 6.1$ \\
\hline \multicolumn{3}{|l|}{ Sex-no. (\%) } \\
\hline Male & $94(75.8)$ & $85(66.9)$ \\
\hline Female & $30(24.2)$ & $42(33.1)$ \\
\hline Duration of Parkinson's disease $-y r$ & $7.3 \pm 3.1$ & $7.7 \pm 2.7$ \\
\hline \multicolumn{3}{|l|}{ Dyskinesiał } \\
\hline No. of patients & 84 & 94 \\
\hline Duration $-y r$ & $1.4 \pm 0.8$ & $1.5 \pm 0.8$ \\
\hline \multicolumn{3}{|l|}{ Motor fluctuations广 } \\
\hline No. of patients & 121 & 124 \\
\hline Duration $-y r$ & $1.6 \pm 0.8$ & $1.8 \pm 0.8$ \\
\hline \multicolumn{3}{|l|}{ Treatment with levodopa } \\
\hline No. of patients & 111 & 115 \\
\hline Duration - yr & $4.8 \pm 3.3$ & $5.0 \pm 3.3$ \\
\hline \multicolumn{3}{|l|}{ Treatment with dopamine agonist } \\
\hline No. of patients & 118 & 115 \\
\hline Duration $-y r$ & $5.9 \pm 3.0$ & $6.1 \pm 3.0$ \\
\hline Levodopa-equivalent daily dose - mg & $918.8 \pm 412.5$ & $966.9 \pm 416.5$ \\
\hline
\end{tabular}

* Plus-minus values are means \pm SD. There were no significant between-group differences.

$\uparrow$ The presence of dyskinesias or fluctuations for 3 years or less was an eligibility criterion.

to treatment, and death during the study period. The per-protocol analysis included 116 patients in the neurostimulation group and 110 in the medical-therapy group (Fig. S1 in the Supplementary Appendix). Baseline characteristics did not differ significantly between the treatment groups (Table 1). The mean duration of Parkinson's disease was 7.5 years, and patients were included in the study a mean of 1.7 years after the onset of levodopa-induced motor complications of any severity.

\section{QUALITY-OF-LIFE, ACTIVITIES OF DAILY LIVING, AND MOTOR OUTCOMES}

The primary outcome (PDQ-39 summary index score) was improved from baseline to 24 months by $26 \%$ in the neurostimulation group but worsened by $1 \%$ in the medical-therapy group (Table 2). In the intention-to-treat population, the betweengroup difference in the mean change from baseline was 8.0 points $(P=0.002)$, which was similar to the between-group differences in the per-protocol population and the group of patients who completed the PDQ-39 assessment. The maximum 


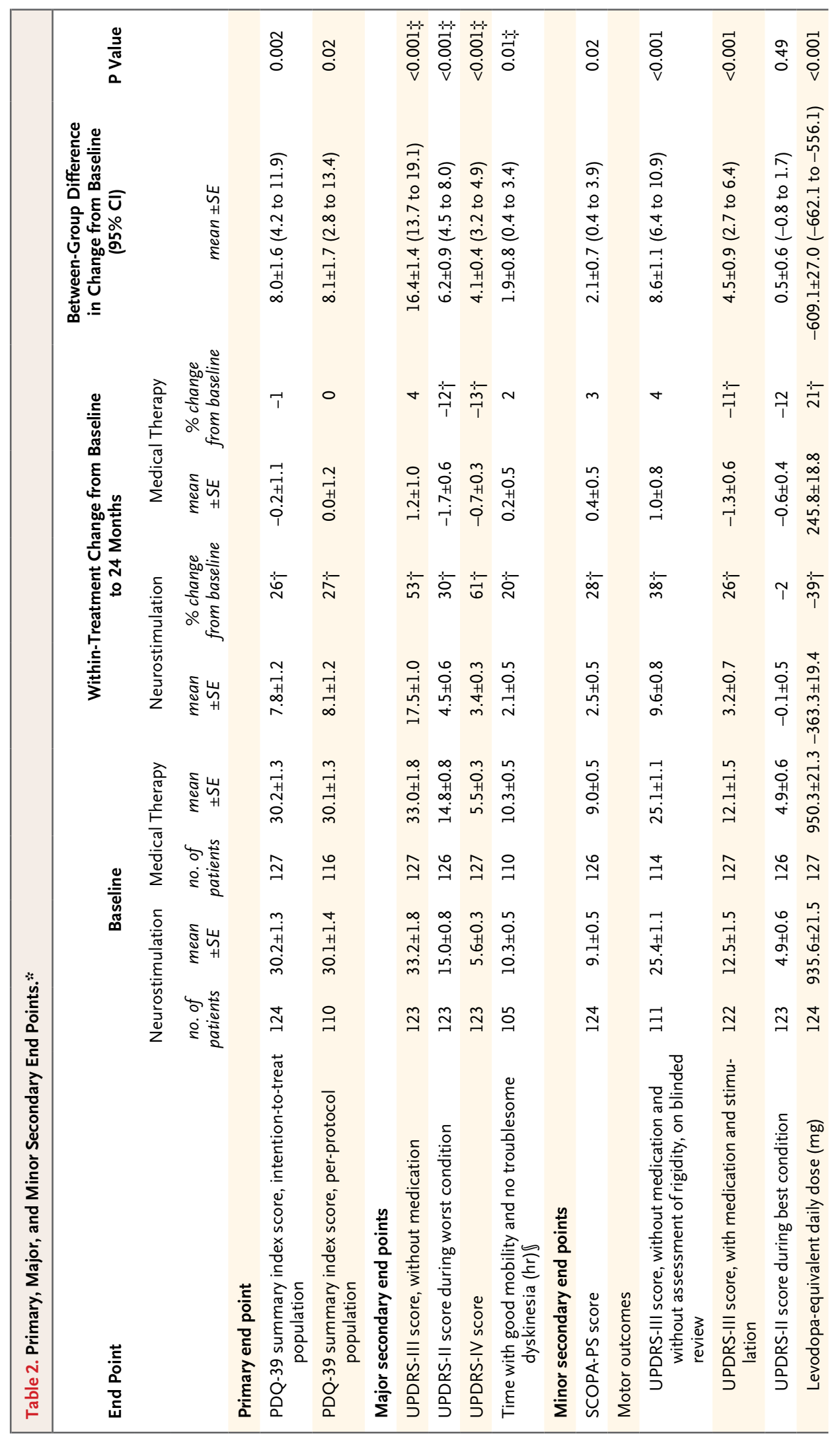




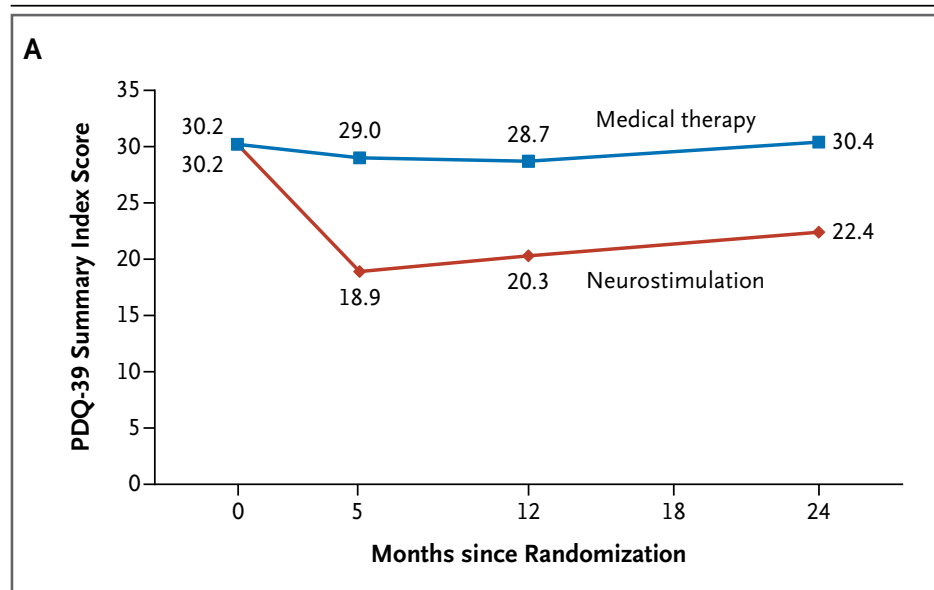

B

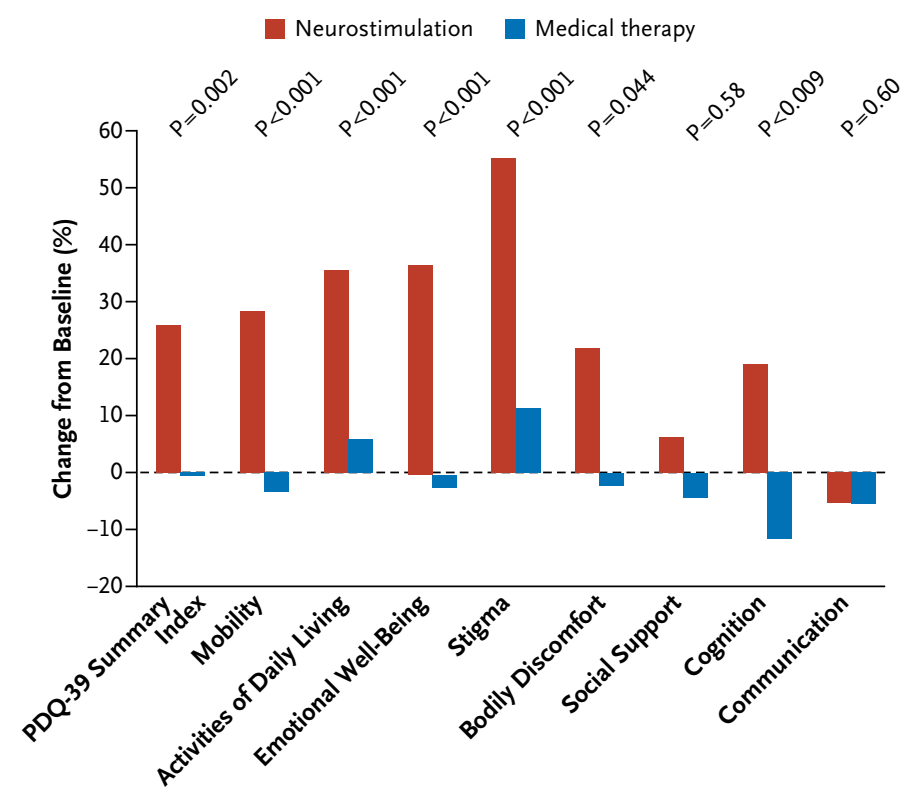

Figure 1. Quality of Life as Assessed by Means of the Parkinson's Disease Questionnaire (PDQ-39).

Panel A shows scores on the summary index of the PDQ-39 at baseline and at 5,12 , and 24 months for both treatment groups. Scores range from 0 to 100 , with lower values indicating better quality of life. Panel B shows the change in the subscores for the various domains of the PDQ-39. Positive values indicate improvement.

effect was reached at 5 months and remained stable for up to 24 months (Fig. 1A). Scores in all domains of the PDQ-39 except for communication and social support showed significant improvement in favor of neurostimulation (Fig. 1B). The SCOPA-PS score for psychosocial performance was also significantly better in the neuro- stimulation group than in the medical-therapy group ( $\mathrm{P}=0.02)$. These changes were confirmed by further testing with the use of generic and disease-specific quality-of-life and disability scales (Table S3 in the Supplementary Appendix).

UPDRS-III scores for the severity of parkinsonian motor signs in the off-medication condition (with assessments conducted after medications had been withheld for $\geq 12$ hours) improved by $53 \%$ in the neurostimulation group; the betweengroup difference in mean change at 2 years was 16.4 points in favor of neurostimulation $(\mathrm{P}<0.001)$. This finding was confirmed by means of blinded assessment of the video recordings of the UPDRSIII score, corroborating the significant improvement among patients in the neurostimulation group, as compared with the medical-therapy group, in which scores did not change $(\mathrm{P}<0.001)$ (Table 2). A smaller but significant benefit was observed for patients in the neurostimulation group in the on-medication and on-stimulation condition (Table 2). UPDRS-IV scores for levodopainduced complications, including motor fluctuations and dyskinesia, improved by $61 \%$ in the neurostimulation group, with a 4.1-point difference between treatment groups $(\mathrm{P}<0.001)$ (Table 2 ), which was confirmed by the scores on a dyskinesia scale (Table S3 in the Supplementary Appendix).

UPDRS-II scores for activities of daily living in the worst condition during the preceding week differed by 6.2 points in favor of neurostimulation $(\mathrm{P}<0.001)$ (Table 2$)$, with no significant between-group difference for the score in the best condition. Time with good mobility and no troublesome dyskinesia, as recorded by the patients in daily diaries, increased by $20 \%$ in the neurostimulation group, with a between-group difference of 1.9 hours $(\mathrm{P}=0.01)$ (Table 2). Time with bad mobility was significantly shortened in the neurostimulation group, with a betweengroup difference of 1.8 hours $(\mathrm{P}=0.006)$ (Table S3 in the Supplementary Appendix). Betweengroup differences in time with troublesome dyskinesia and sleep time (Fig. S2 in the Supplementary Appendix) were not significant.

Medication use was significantly changed in both treatment groups. The levodopa-equivalent daily dose was reduced by $39 \%$ in the neurostimulation group but was increased by $21 \%$ in the medical-therapy group, with a between-group 
difference of $609 \mathrm{mg}(\mathrm{P}<0.001)$ (Table 2, and Table S3 in the Supplementary Appendix).

\section{COGNITIVE AND EMOTIONAL OUTCOMES}

No significant between-group differences were observed for cognitive assessments obtained with the use of the Mattis Dementia Rating Scale or the UPDRS-I (Table 2). Changes in mood, as rated by the examiner (score on the Montgomery and Åsberg Depression Rating Scale) or by patients (score on the Beck Depression Inventory II), were in favor of neurostimulation, as were the scores on the Brief Psychiatric Rating Scale for overall psychiatric morbidity. Scores on the Starkstein Apathy Scale worsened in both treatment groups, with a nonsignificant trend toward worse ratings for apathy among patients in the neurostimulation group than among those in the medicaltherapy group (Table 2).

\section{ADVERSE EVENTS}

A total of 68 patients in the neurostimulation group and 56 in the medical-therapy group had at least one serious adverse event. Numbers of all adverse events were similar in the two groups (Table 3, and Table S4 in the Supplementary Appendix). Two patients in the neurostimulation group and 1 in the medical-therapy group committed suicide; these were the only deaths. Suicidal ideation and suicide attempts were of similar frequency in the two groups, but depression was more frequent in the neurostimulation group. Serious adverse events related to motor problems, impulse control disorders, and psychotic manifestations were more common in the medical-therapy group. Of 26 serious adverse events related to surgery or the implanted device, including a brain abscess and a case of unspecific edema, all but 1 resolved completely; the exception was a case of impaired wound healing, which resulted in mild scarring. Unscheduled visits were offered for all health problems; 343 visits occurred in the medical-therapy group and 277 in the neurostimulation group.

\section{ASSESSED CONSISTENCY OF TREATMENT WITH GUIDELINES}

Experts assessed medical therapy as consistent with guidelines in 120 patients $(96.8 \%)$ in the neurostimulation group and in 120 patients $(94.5 \%)$ in the medical-therapy group. Stimula- tion parameters at 24 months were similar for both hemispheres, with a mean $( \pm S D)$ stimulation strength of $2.8 \pm 0.7 \mathrm{~V}$, a mean stimulation frequency of $142 \pm 27 \mathrm{~Hz}$, and a mean pulse duration of $66 \pm 13 \mu$ s (pooled data).

\section{DISCUSSION}

This patient population with relatively mild parkinsonian motor signs differed from the populations in previous controlled studies of neurostimulation: the mean duration of disease in the patients in our study was only 7.5 years, as compared with 11.1 to 13.8 years $^{7-10}$; patients were younger (52 years, as compared with 59 to 62 years $\left.{ }^{8-11}\right)$; and fluctuations and dyskinesia were present for only 1.7 and 1.5 years, respectively. Nevertheless, patients in the neurostimulation group had a $26 \%$ improvement in the PDQ-39 summary index score, corroborating the $24 \%$ improvement observed in our pilot study ${ }^{15}$ and the $25 \%$ improvement observed in our large trial involving patients with advanced Parkinson's disease, ${ }^{7}$ in both of which the PDQ-39 summary index score was the primary outcome. The between-group difference in the mean change from baseline of 8.0 points for the PDQ-39 summary index score in favor of neurostimulation is a clinically relevant finding. ${ }^{33-35}$ The profile of improvement in subdomains of quality of life differed slightly from that among patients treated at an advanced stage of the disease, ${ }^{7}$ because activity of daily living, emotional well-being, and cognition improved more during this study than during prior studies involving patients with advanced Parkinson's disease. The improvement in the primary outcome was consistent with other measures of quality of life, psychiatric morbidity, and psychosocial function, which all improved in the neurostimulation group, as compared with the medical-therapy group.

Patients received neurostimulation at a stage of the disease when medical treatment is still effective for motor function, as reflected by several findings in the medical-therapy group: the absence of a significant change from baseline in the UPDRS-III motor score and only minimal worsening in the UPDRS-II score for activities of daily living and in the UPDRS-IV score for levodopainduced complications. In addition, diary results for mobility did not change significantly from 
baseline in the medical-therapy group, but time with bad mobility was reduced and time with good mobility was significantly increased in the neurostimulation group. The observed difference between the treatment groups is thus due to an improvement among patients receiving neurostimulation, as compared with those receiving medical therapy alone, not to deterioration among the latter patients.

Neurostimulation in combination with medical therapy can therefore improve motor symptoms better than medical therapy alone at this earlier stage. Because the levodopa response predicts the extent of the effect of subthalamic stimulation on parkinsonian motor signs, the improvement of the off-medication condition with the use of stimulation was expected. More- over, in spite of a ceiling effect, the combination of stimulation and medication resulted in a small but significant improvement in the motor score, as compared with medical treatment alone. As a main benefit from the patient's perspective, activities of daily living were improved among patients with neurostimulation in the worst condition during the day, although, as expected, there was no significant difference between groups in the best condition.

Overall, adverse events were more frequent among patients receiving neurostimulation than among those receiving medical therapy only. However, this result was mainly due to a higher number of mild adverse events in the neurostimulation group than in the medical-therapy group. Serious adverse events in the medical-therapy

\begin{tabular}{|c|c|c|c|c|}
\hline \multirow[t]{2}{*}{ Event } & \multicolumn{2}{|c|}{ Neurostimulation ( $\mathrm{N}=124$ ) } & \multicolumn{2}{|c|}{ Medical Therapy $(\mathrm{N}=127)$} \\
\hline & $\begin{array}{l}\text { no. of } \\
\text { events }\end{array}$ & $\begin{array}{l}\text { no. of patients } \\
\text { with event (\%) }\end{array}$ & $\begin{array}{l}\text { no. of } \\
\text { events }\end{array}$ & $\begin{array}{l}\text { no. of patients } \\
\text { with event (\%) }\end{array}$ \\
\hline Serious adverse events & 123 & $68(54.8)$ & 128 & $56(44.1)$ \\
\hline Death, all by suicide & 2 & $2(1.6)$ & 1 & $1(0.8)$ \\
\hline Life-threatening event & 14 & $12(9.7)$ & 17 & $9(7.1)$ \\
\hline Event related to medication or stimulation & 24 & $24(19.4)$ & 52 & 38 (29.9) \\
\hline Worsening of mobility & 5 & $5(4.0)$ & 13 & $11(8.7)$ \\
\hline Motor fluctuations & 0 & 0 & 7 & $7(5.5)$ \\
\hline Dyskinesia & 1 & $1(0.8)$ & 2 & $2(1.6)$ \\
\hline Psychosis or hallucinations & 0 & 0 & 8 & $6(4.7)$ \\
\hline Anxiety & 0 & 0 & 3 & $2(1.6)$ \\
\hline Impulse control disorder & 1 & $1(0.8)$ & 5 & $5(3.9)$ \\
\hline Depression & 6 & $6(4.8)$ & 3 & $1(0.8)$ \\
\hline Suicidal ideation & 1 & $1(0.8)$ & 0 & 0 \\
\hline Suicide attempt & 2 & $2(1.6)$ & 2 & $2(1.6)$ \\
\hline Cardiac disorder & 0 & 0 & 2 & $2(1.6)$ \\
\hline Injury & 3 & $3(2.4)$ & 0 & 0 \\
\hline Respiratory or thoracic disorder & 1 & $1(0.8)$ & 0 & 0 \\
\hline Other & 4 & $4(3.2)$ & 7 & $5(3.9)$ \\
\hline Event related to surgery or device & 26 & $22(17.7)$ & - & - \\
\hline Impaired wound healing & 4 & $4(3.2)$ & - & - \\
\hline Intracerebral abscess or edema & 2 & $2(1.6)$ & - & - \\
\hline Dislocation of devicet & 5 & $4(3.2)$ & - & - \\
\hline Reoperation necessary $\int$ & 4 & $2(1.6)$ & - & - \\
\hline Other & 11 & $10(8.1)$ & - & - \\
\hline Event related to Parkinson's disease & 57 & $39(31.5)$ & 58 & $31(24.4)$ \\
\hline
\end{tabular}




\begin{tabular}{|c|c|c|c|c|}
\hline \multirow[t]{2}{*}{ Event } & \multicolumn{2}{|c|}{ Neurostimulation $(\mathrm{N}=124)$} & \multicolumn{2}{|c|}{ Medical Therapy $(\mathrm{N}=127)$} \\
\hline & $\begin{array}{l}\text { no. of } \\
\text { events }\end{array}$ & $\begin{array}{l}\text { no. of patients } \\
\text { with event (\%) }\end{array}$ & $\begin{array}{l}\text { no. of } \\
\text { events }\end{array}$ & $\begin{array}{l}\text { no. of patients } \\
\text { with event (\%) }\end{array}$ \\
\hline Adverse events & 1032 & $121(97.6)$ & 925 & $125(98.4)$ \\
\hline Mild & 636 & $105(84.7)$ & 435 & $100(78.7)$ \\
\hline Moderate & 364 & $95(76.6)$ & 437 & $98(77.2)$ \\
\hline Severe & 32 & $20(16.1)$ & 53 & $35(27.6)$ \\
\hline \multicolumn{5}{|l|}{ Moderate or severe\| } \\
\hline Dyskinesia & 24 & $18(14.5)$ & 69 & $49(38.6)$ \\
\hline Gait disorder & 25 & $17(13.7)$ & 25 & $15(11.8)$ \\
\hline Worsening of mobility & 74 & $40(32.3)$ & 137 & $72(56.7)$ \\
\hline Depression & 27 & $21(16.9)$ & 32 & $23(18.1)$ \\
\hline Sleep disorder & 17 & $14(11.3)$ & 19 & $16(12.6)$ \\
\hline Impulse control disorder & 26 & $18(14.5)$ & 23 & $15(11.8)$ \\
\hline Musculoskeletal or connective tissue disorder & 14 & $13(10.5)$ & 14 & $11(8.7)$ \\
\hline Pain & 30 & $19(15.3)$ & 15 & $12(9.4)$ \\
\hline Weight change & 15 & $15(12.1)$ & 6 & $6(4.7)$ \\
\hline
\end{tabular}

* All adverse events are listed according to the Medical Dictionary for Regulatory Activities, version 14.1.

$\dagger$ Worsening of mobility was defined as tremor, rigidity, akinesia, wearing off of medication effect, dystonia, or worsening of symptoms of Parkinson's disease.

$\checkmark$ Dislocation of device was defined as the dislocation of the stimulator, cable, or lead.

$\int$ Reoperation was necessary in order to repair the stimulator or lead.

All nonserious adverse events are listed.

|| Data include moderate, severe, or life-threatening adverse events that were not serious and were reported in at least $10 \%$ of the patients in at least one group. These latter adverse events were judged to be life-threatening by the investigator but did not fulfill the criteria for seriousness, which include hospitalization, prolongation of hospitalization, or death. There were nonlethal life-threatening adverse events that did not lead to hospitalization. The remaining moderate, severe, and life-threatening events are reported in detail in Table S4 in the Supplementary Appendix. A moderate adverse event was defined as one that might interfere with normal activity and lead to the consideration of medical intervention or close followup, and a severe adverse event as an event posing a substantial risk to the patient's health and likely to require medical intervention or close follow-up. Severe adverse events reported here included two life-threatening adverse events that were not judged to fulfill the criteria for seriousness.

group were more often related to problems of mobility and side effects of medications (hallucinations and behavioral problems), whereas major depression occurred more often among patients with neurostimulation, despite an overall improvement in mood at the end of the trial. The frequency of suicidal behavior, including suicide, was high ${ }^{36}$ but did not differ between treatment groups. Although there were three suicides (two in the neurostimulation group and one in the medical-therapy group) as well as four suicide attempts (two in the neurostimulation group and two in the medical-therapy group) during the study, this trial did not suggest that neurostimulation is associated with a higher risk of suicide than medical therapy. Instead, we hypothesize that the decision to eventually undergo neuro- stimulation may select a specific subgroup of patients with a higher risk for suicidal behavior than the general population. The monitoring procedures established in this study may be useful in the future. A total of 26 serious adverse events were related directly to surgery or the implanted devices; 25 of them resolved completely, and 1 left a cutaneous scar.

The strengths of this study include the strict standards for interventions, close monitoring of side effects, the small number of withdrawals, and the consistency between the results of the intention-to-treat and per-protocol analyses. Because standards of medical therapy were well respected in both treatment groups and because motor symptoms and quality of life did not change in the medical-therapy group, the improvement 
among patients who underwent surgical implantation can be attributed to neurostimulation. To overcome the difficulties of blinding in neurostimulation studies, ${ }^{21}$ we introduced the blinded review of motor scores with the use of video recordings, which confirmed the superior effect of neurostimulation over medical therapy, supporting the validity of the main study result.

In conclusion, we found that neurostimulation was superior to medical therapy alone at a relatively early stage of Parkinson's disease, before the appearance of severe disabling motor complications. Neurostimulation may be a therapeutic option for patients at an earlier stage than current recommendations suggest. ${ }^{23,37}$

Supported by grants from the German Ministry of Research (Klinische Studien 01KG0502) and the French Programme Hospitalier de Recherche Clinique National (P050909) and by Medtronic.

Dr. Schuepbach reports receiving consulting fees and lecture fees from Medtronic and serving as an expert witness for Lundbeck. Dr. Knudsen reports receiving lecture fees and payment for the development of educational programs from Medtronic. Dr. Volkmann reports receiving grant support, lecture fees, and consulting fees from Medtronic. Dr. Krack reports receiving grant support from Medtronic. Dr. Timmermann reports receiving grant support from Medtronic; lecture fees from Medtronic, Teva Pharmaceuticals, Lundbeck, Union Chimique Belge (UCB), Bayer HealthCare, Meda Pharmaceuticals, Orion, GlaxoSmithKline, Boehringer Ingelheim, Desitin, Gianni Public Relations, Eumecom, Cephalon, Abbott Laboratories, and GE HealthCare; consulting fees from Bayer HealthCare, Teva Pharmaceuticals, and UCB; and payment for board membership from Medtronic, UCB, and Teva Pharmaceuticals and for preparation of a manuscript from Teva Pharmaceuticals. Dr. Hesekamp reports receiving travel support from Lundbeck, Bayer Schering Pharma, Boehringer Ingelheim, and UCB. Dr. Falk reports receiving lecture fees from Medtronic. Dr. Mehdorn reports receiving consulting fees, lecture fees, grant support, travel support, and payment for the development of educational presentations from Medtronic. Dr. Paschen reports receiving lecture fees from Medtronic and travel support from Ipsen and Talecris. Dr. Maarouf reports receiving consulting fees, lecture fees, grant support, and travel support from Medtronic. Dr. Barbe reports receiving grant support from Medtronic and lecture fees from Medtronic and GE HealthCare. Dr. Fink reports receiving grant support from Medtronic; consulting fees from
Boehringer Ingelheim; lecture fees from Teva Pharmaceuticals, GlaxoSmithKline, Boehringer Ingelheim, and Desitin; and royalties from Thieme Medical Publishers. Dr. Kupsch reports receiving lecture fees from Allergan, Boehringer Ingelheim, Ipsen, Lundbeck, Medtronic, Merck, Merz, Orion, and UCB and travel support from Ipsen. Dr. Schneider reports receiving lecture fees from Medtronic. Dr. Ory-Magne reports receiving payment for board membership from Novartis and Abbott Laboratories and lecture fees from Boehringer Ingelheim, Aguettant, and UCB. Dr. Brefel Courbon reports receiving payment for board membership from Lundbeck and Novartis. Dr. Vesper reports receiving consulting fees and payment for the development of educational materials from Medtronic. Dr. Schnitzler reports receiving grant support and lecture fees from Medtronic. Dr. Wojtecki reports receiving consulting fees and lecture fees from Medtronic. Dr. Houeto reports receiving consulting fees and payment for development of educational presentations from Medtronic. Dr. Damier reports receiving payment for board membership from Lundbeck and Teva Pharmaceuticals. Dr. Sixel-Doering reports receiving payment for board membership from Orion and UCB and lecture fees from Boehringer Ingelheim, Cephalon, GlaxoSmithKline, Meda Pharmaceuticals, Medtronic, Novartis, and UCB. Dr. Gharabaghi reports receiving grant support, lecture fees, and travel support from Medtronic. Dr. Krüger reports receiving lecture fees from Medtronic and travel support from Abbott Laboratories, UCB, and Cephalon. Dr. Pinsker reports receiving travel support from Medtronic. Dr. Amtage reports receiving travel support from UCB, Medtronic, Merz, and Orion. Dr. Thobois reports receiving payment for board membership from Novartis; consulting fees from UCB, Novartis, and GlaxoSmithKline; grant support from Eutherapie; and payment for the development of educational presentations from Boehringer Ingelheim. Dr. Mertens reports receiving consulting fees, lecture fees, and travel support from Medtronic. Dr. Hartmann reports receiving consulting fees from BioProject. Dr. Oertel reports receiving payment for board membership from Merck Sharp \& Dohme, UCB, and Novartis and lecture fees from Roche, Novartis, Orion, Desitin, Boehringer Ingelheim, and GlaxoSmithKline, and holding stock in Roche. Dr. Post reports receiving grant support from Medtronic and Abbott Laboratories. Dr. Speelman reports receiving consulting fees from Medtronic and Ipsen. Dr. Deuschl reports receiving payment for board membership from Teva Pharmaceuticals and Novartis; lecture fees from Teva Pharmaceuticals, Lundbeck, and Medtronic; and royalties from Thieme Medical Publishers. No other potential conflict of interest relevant to this article was reported.

Disclosure forms provided by the authors are available with the full text of this article at NEJM.org.

We thank all participating patients, monitors, project managers, and data managers.

APPENDIX

The authors' full names and degrees are as follows: W.M. Michael Schuepbach, M.D., Joern Rau, M.Sc., Karina Knudsen, M.D., Jens Volkmann, M.D., Ph.D., Paul Krack, M.D., Ph.D., Lars Timmermann, M.D., Ph.D., Thomas D. Hälbig, M.D., Helke Hesekamp, M.D., Soledad M. Navarro, M.D., Niklaus Meier, M.D., Daniela Falk, M.D., Maximilian Mehdorn, M.D., Ph.D., Steffen Paschen, M.D., Mohammad Maarouf, M.D., Michael T. Barbe, M.D., Gereon R. Fink, M.D., Ph.D., Andreas Kupsch, M.D., Ph.D., Doreen Gruber, M.D., Gerd-H. Schneider, M.D., Eric Seigneuret, M.D., Andrea Kistner, Ph.D., Patrick Chaynes, M.D., Ph.D., Fabienne Ory-Magne, M.D., Christine Brefel Courbon, M.D., Jan Vesper, M.D., Ph.D., Alfons Schnitzler, M.D., Ph.D., Lars Wojtecki, M.D., Jean-Luc Houeto, M.D., Ph.D., Benoît Bataille, M.D., David Maltête, M.D., Ph.D., Philippe Damier, M.D., Ph.D., Sylvie Raoul, M.D., Ph.D., Friederike SixelDoering, M.D., Dieter Hellwig, M.D., Ph.D., Alireza Gharabaghi, M.D., Ph.D., Rejko Krüger, M.D., Ph.D., Marcus O. Pinsker, M.D., Ph.D., Florian Amtage, M.D., Jean-Marie Régis, M.D., Ph.D., Tatiana Witjas, M.D., Stephane Thobois, M.D., Ph.D., Patrick Mertens, M.D., Ph.D., Manja Kloss, M.D., Andreas Hartmann, M.D., Wolfgang H. Oertel, M.D., Ph.D., Bart Post, M.D., Ph.D., Hans Speelman, M.D., Ph.D., Yves Agid, M.D., Ph.D., Carmen Schade-Brittinger, B.Sc., and Günther Deuschl, M.D., Ph.D.

The authors' affiliations are as follows: Assistance Publique-Hôpitaux de Paris, Centre d'Investigation Clinique (CIC) 9503, Institut du Cerveau et de la Moelle Épinière, Département de Neurologie, Université Pierre et Marie Curie-Paris 6 and INSERM, Centre Hospitalier Universitaire (CHU) Pitié-Salpêtrière (W.M.M.S., T.D.H., H.H., N.M., A.H., Y.A.), and the Department of Neurosurgery, 
CHU Pitié-Salpêtrière (S.M.N.), Paris; the Departments of Neurology (P.K., A. Kistner) and Neurosurgery (E.S.), University Hospital, INSERM Unité 836, Grenoble; the Department of Neurology, Neurosurgery, and Pharmacology, University Hospital, Toulouse (P.C., F.O.-M., C.B.C.); Service de Neurologie, CIC INSERM-0802 INSERM Unité 1084, Laboratoire de Neurosciences Expérimentales et Cliniques (J.-L.H.), and the Department of Neurosurgery (B.B.), Universite de Poitiers, Poitiers; the Department of Neurology, University Hospital, Rouen (D.M.); CIC, CHU Nantes, Nantes (P.D., S.R.); the Department of Functional Neurosurgery, Aix-Marseille University (J.-M.R.), and the Department of Neurology, University Hospital Timone (T.W.), Marseille; University Lyon 1, Hospices Civils de Lyon, Hôpital Neurologique Pierre Wertheimer, Neurologie C (S.T.), and the Department of Neurosurgery, University Hospital (P.M.), Lyon - all in France; the Coordinating Center for Clinical Trials (J.R., C.S.-B.) and the Departments of Neurosurgery (D.H.) and Neurology (W.H.O.), Philipps University, Marburg; the Departments of Neurology (K.K., J. Volkmann, S.P., G.D.) and Neurosurgery (D.F., M. Mehdorn), University Hospital Schleswig-Holstein, Kiel; the Departments of Neurology (L.T., M.T.B., G.R.F.) and Neurosurgery (M. Maarouf), University of Cologne, Cologne; the Departments of Neurology (A. Kupsch, D.G.) and Neurosurgery (G.-H.S.), Charité Campus Virchow Hospital, Berlin; the Departments of Neurosurgery (J. Vesper) and Neurology, Institute of Clinical Neuroscience and Medical Psychology (A.S., L.W.), Heinrich-Heine University, Duesseldorf; Paracelsus-ElenaKlinik, Kassel (F.S.-D.); the Department of Neurosurgery (A.G.) and Center of Neurology and Hertie Institute for Clinical Brain Research (R.K.), University Hospital, Tuebingen; the Departments of Neurosurgery (M.O.P.) and Neurology (F.A.), University Hospital, Freiburg; and the Department of Neurology, University Hospital, Heidelberg (M.K.) - all in Germany; and the Department of Neurology, Radboud University Nijmegen Medical Center, Nijmegen (B.P.); and the Department of Neurology, University of Amsterdam, Amsterdam (H.S.) — both in the Netherlands.

\section{REFERENCES}

1. Vidailhet M. Movement disorders in 2010: Parkinson disease - symptoms and treatments. Nat Rev Neurol 2011; 7:70-2.

2. Schrag A, Jahanshahi M, Quinn N How does Parkinson's disease affect quality of life? A comparison with quality of life in the general population. Mov Disord 2000;15:1112-8.

3. Schrag A, Spottke A, Quinn NP, Dodel R. Comparative responsiveness of Parkin son's disease scales to change over time. Mov Disord 2009;24:813-8.

4. Post B, Muslimovic D, van Geloven N, Speelman JD, Schmand B, de Haan RJ. Progression and prognostic factors of motor impairment, disability and quality of life in newly diagnosed Parkinson's disease. Mov Disord 2011;26:449-56.

5. Limousin P, Krack P, Pollak P, et al. Electrical stimulation of the subthalamic nucleus in advanced Parkinson's disease. N Engl J Med 1998;339:1105-11.

6. Kleiner-Fisman G, Herzog J, Fisman DN, et al. Subthalamic nucleus deep brain stimulation: summary and meta-analysis of outcomes. Mov Disord 2006;21:Suppl 14:S290-S304.

7. Deuschl G, Schade-Brittinger C, Krack P, et al. A randomized trial of deepbrain stimulation for Parkinson's disease. N Engl J Med 2006;355:896-908. [Erratum, N Engl J Med 2006;355:1289.]

8. Williams A, Gill S, Varma T, et al. Deep brain stimulation plus best medical therapy versus best medical therapy alone for advanced Parkinson's disease (PD SURG trial): a randomised, open-label trial. Lancet Neurol 2010;9:581-91.

9. Follett KA, Weaver FM, Stern M, et al. Pallidal versus subthalamic deep-brain stimulation for Parkinson's disease. N Engl J Med 2010;362:2077-91.

10. Okun MS, Gallo BV, Mandybur G, et al. Subthalamic deep brain stimulation with a constant-current device in Parkinson's disease: an open-label randomised controlled trial. Lancet Neurol 2012;11: 140-9. [Erratum, Lancet Neurol 2012; 11:208.]

11. Krack P, Batir A, Van Blercom N, et al. Five-year follow-up of bilateral stimulation of the subthalamic nucleus in advanced Parkinson's disease. N Engl J Med 2003;349:1925-34.

12. Fasano A, Romito LM, Daniele A, et al. Motor and cognitive outcome in patients with Parkinson's disease 8 years after subthalamic implants. Brain 2010; 133:2664-76.

13. Moro E, Lozano AM, Pollak P, et al. Long-term results of a multicenter study on subthalamic and pallidal stimulation in Parkinson's disease. Mov Disord 2010; 25:578-86.

14. Marinus J, Visser M, Martínez-Martín P, van Hilten JJ, Stiggelbout AM. A short psychosocial questionnaire for patients with Parkinson's disease: the SCOPA-PS. J Clin Epidemiol 2003;56:61-7.

15. Schüpbach WM, Maltête D, Houeto JL, et al. Neurosurgery at an earlier stage of Parkinson disease: a randomized, controlled trial. Neurology 2007;68:267-71.

16. Hoehn MM, Yahr MD. Parkinsonism: onset, progression and mortality. Neurology 1967;17:427-42.

17. Fahn S, Elton RL. Unified Parkinson's Disease Rating Scale. In: Fahn S, Marsden CD, Calne D, Goldstein M, eds. Recent developments in Parkinson's disease. Florham Park, NJ: MacMillan Health Care Information, 1987:153-63.

18. Goldman HH, Skodol AE, Lave TR. Revising axis V for DSM-IV: a review of measures of social functioning. Am J Psychiatry 1992;149:1148-56.

19. Mattis S. Mental status examination for organic mental syndrome in the elderly patient. In: Bellak L, Karasu T, eds. Geriatric psychiatry. New York: Grune and Stratton, 1976:77-121.

20. Beck AT, Ward $\mathrm{CH}$, Mendelson $\mathrm{M}$, Mock J, Erbaugh J. An inventory for mea- suring depression. Arch Gen Psychiatry 1961;4:561-71.

21. Deuschl G, Schüpbach WMM, Knudsen $\mathrm{K}$, et al. Stimulation of the subthalamic nucleus at an earlier disease stage of Parkinson's disease: concept and standards of the EARLYSTIM-study. Parkinsonism Relat Disord 2013;19:56-61.

22. Post B, Merkus MP, de Bie RM, de Haan RJ, Speelman JD. Unified Parkinson's Disease Rating Scale motor examination: are ratings of nurses, residents in neurology, and movement disorders specialists interchangeable? Mov Disord 2005;20:1577-84.

23. Horstink M, Tolosa E, Bonuccelli U, et al. Review of the therapeutic management of Parkinson's disease - report of a joint task force of the European Federation of Neurological Societies (EFNS) and the Movement Disorder Society-European Section (MDS-ES). Part II: late (complicated) Parkinson's disease. Eur J Neurol 2006;13:1186-202.

24. Fitzpatrick R, Peto V, Jenkinson C, Greenhall R, Hyman N. Health-related quality of life in Parkinson's disease: a study of outpatient clinic attenders. Mov Disord 1997;12:916-22.

25. Martinez-Martin P, Jeukens-Visser M, Lyons KE, et al. Health-related quality-oflife scales in Parkinson's disease: critique and recommendations. Mov Disord 2011; 26:2371-80.

26. Flemenbaum A, Zimmermann RL. Inter- and intra-rater reliability of the Brief Psychiatric Rating Scale. Psychol Rep 1973;32:783-92.

27. Montgomery SA, Åsberg M. A new depression scale designed to be sensitive to change. Br J Psychiatry 1979;134:382-9.

28. Starkstein SE, Mayberg HS, Preziosi TJ, Andrezejewski P, Leiguarda R, Robinson RG. Reliability, validity, and clinical correlates of apathy in Parkinson's disease. J Neuropsychiatry Clin Neurosci $1992 ; 4: 134-9$ 
29. Tomlinson CL, Stowe R, Patel S, Rick C, Gray R, Clarke CE. Systematic review of levodopa dose equivalency reporting in Parkinson's disease. Mov Disord 2010; 25:2649-53.

30. Mallinckrodt $\mathrm{CH}$, Clark WS, David SR. Accounting for dropout bias using mixed-effects models. J Biopharm Stat 2001;11:9-21.

31. Beunckens C, Molenberghs G, Kenward M. Direct likelihood analysis versus simple forms of imputation for missing data in randomized clinical trials. Clin Trials 2005;2:379-86.

32. Hochberg Y. A sharper Bonferroni procedure for multiple tests of significance. Biometrika 1988;75:800-2.

33. Smeding HM, Speelman JD, Huizenga HM, Schuurman PR, Schmand B. Predictors of cognitive and psychosocial outcome after STN DBS in Parkinson's disease. J Neurol Neurosurg Psychiatry 2011; 82:754-60.

34. Daniels C, Krack P, Volkmann J, et al. Is improvement in the quality of life after subthalamic nucleus stimulation in Parkinson's disease predictable? Mov Disord 2011;26:2516-21.

35. Peto V, Jenkinson C, Fitzpatrick R. Determining minimally important differ- ences for the PDQ-39 Parkinson's disease questionnaire. Age Ageing 2001;30:299302.

36. Voon V, Krack P, Lang AE, et al. A multicentre study on suicide outcomes following subthalamic stimulation for Parkinson's disease. Brain 2008;131:27208.

37. Fox SH, Katzenschlager R, Lim SY, et al. The Movement Disorder Society evidence-based medicine review update: treatments for the motor symptoms of Parkinson's disease. Mov Disord 2011;26: Suppl 3:S2-S41.

Copyright (C) 2013 Massachusetts Medical Society. 